\title{
Multiperiodicity in pulsating red giants
}

\author{
J.R. Percy, A.G. Bakos, G. Besla, D. Hou, V. Velocci \\ Department of Astronomy $\&$ Astrophysics, University of Toronto, \\ Toronto, ON Canada M5S $3 \mathrm{H} 8$ \\ G.W. Henry \\ Center of Excellence in Information Systems, Tennessee State \\ University, Nashville TN 37203-3401, USA
}

\begin{abstract}
We describe several aspects of the variability of bright pulsating red giant (PRG) stars, with emphasis on the determination of pulsation modes and pulsation masses by using observed multiperiodicity and pulsation constants ( $Q$-values). Our studies of bright PRGs provide a model for the analysis and interpretation of the thousands of PRGs being discovered in surveys of Local Group galaxies.
\end{abstract}

\section{Introduction}

Red giants cooler than spectral type K5III are variable in brightness. Such stars make up about 10 per cent of the stars in the Bright Star Catalogue. They have temperatures of $4000 \mathrm{~K}$ and cooler, radii of $25 \mathrm{R}_{\odot}$ and larger, and masses which are typically solar. The $V$ amplitude of variability ranges from millimagnitudes at K5III to many magnitudes in the rarest, coolest Mira stars. The periods range from 10 to over $200 \mathrm{~d}$, and are consistent with low-order radial pulsation. High-overtone periods are unlikely (Percy \& Hosick 2002), despite earlier claims (Koen \& Laney 2000). About a third of PRGs have long secondary periods, whose nature and cause are unknown. This paper deals with small-amplitude PRGs, which are by far the most numerous kind. They are sometimes classified as "semi-regular" or "irregular", but we find that many of them are nearly periodic, or are multiperiodic (Percy, Wilson \& Henry 2001; Percy et al. 2003).

\section{Data and Analysis}

Our data come primarily from the American Association of Variable Star Observers (AAVSO) Photoelectric Photometry Program (Percy et al. 1996) and from Automated Photometric Telescopes (APTs) operated by Tennessee State University (Percy, Wilson \& Henry 2001). As described in those papers, periods are determined by a combination of light curve, Fourier, and self-correlation analysis. In several multiperiodic PRGs, the periods are derived from individual seasons of data using the least-squares program Period98 (Sperl 1998) in com- 
bination with Fourier analysis of the full dataset. Sample light curves of PRGs are shown by Percy, Wilson \& Henry (2001).

\section{Pulsation Mode Determination from $Q$-Values}

The pulsation constant $Q$ is defined as the period in days, times the square root of the average density of the star in solar units. Observed $Q$-values can be determined from the observed period, from observed or deduced radii, and from a "guesstimate" of the mass (there are no PRGs with directly-determined masses). We have a sample of 77 class-III PRGs. Periods were determined as described above. Temperatures were determined from calibrations of the spectral type and of near-IR colour indices. Luminosities were determined from average magnitudes from the light curves, HIPPARCos parallaxes, and bolometric corrections. Radii were determined from temperatures and luminosities, from calibrations of radius with spectral type or near-IR colour index or, in a few cases, directly from angular diameters. The masses were estimated to be 1.0 to $1.5 \mathrm{M}_{\odot}$; Dumm \& Schild (1998) provide a possible relation between mass and radius for red giants. The observed $Q$-values were compared with theoretical $Q$-values determined by Fox \& Wood (1982), Ostlie \& Cox (1986), and Xiong, Deng \& Cheng (1998). See Percy \& Bakos (2003) for details of the procedures.

Comparison between observed and theoretical $Q$-values suggests that, in about 30 per cent of the stars, the dominant mode is the fundamental mode, in about 50 per cent, it is the first overtone mode, and in about 10 per cent, it is the second overtone mode. There is no correlation between pulsation mode and temperature.

\section{Multiperiodicity in Pulsating Red Giants}

Percy et al. (2003) discovered and analyzed multiperiodicity in five bright PRGs: RZ Ari, V523 Mon, BC CMi, UX Lyn, and FS Com. Each of these stars has two or three periods which recur from season to season, and which can be interpreted as low-order radial pulsation periods. The amplitudes of the modes vary on time scales of $2000-3500 \mathrm{~d}$. We have now identified and analyzed 7 more multiperiodic PRGs: EG And, 4 Ori, UW Lyn, $\mu$ Gem, V642 Her, R Lyr, and $\delta$ Sge. However, 4 Ori is classed as S type, and $\delta$ Sge is luminosity class II, so we do not trust our calibrations of temperature and radius for these. All of these PRGs were selected because their seasonal light curves appeared to be multiperiodic, and their periods were short enough (generally less than $50 \mathrm{~d}$ ) so that they could be determined from individual 200-d seasons of data.

The results of our analysis of these 10 class-III stars are given in Table 1. The pulsation modes have been determined from both the $Q$-values and by comparing the observed period ratios with theoretical ones. Once the pulsation modes have been identified, the observed periods and radii and the theoretical $Q$-values can be used to estimate the "pulsation mass". The pulsation masses are listed in the last column of Table 1. "Rank" refers to the relative amplitude of the mode.

These pulsation masses should be interpreted with caution. They are affected by any error in the observed periods and radii. The grids of theoreti- 
Table 1. Multiperiodic PRGs: Pulsation Modes and Masses.

\begin{tabular}{|c|c|c|c|c|c|c|c|}
\hline Star & $T_{e}(\mathrm{~K})$ & $\mathbf{R} / \mathbf{R}_{\odot}$ & $P(d)$ & Rank & Mode & P ratio & $\mathrm{M} / \mathrm{M}_{\odot}$ \\
\hline EG And & 3711 & 60 & $\begin{array}{l}29.1 \\
47.6\end{array}$ & $\begin{array}{l}1 \\
2\end{array}$ & $\begin{array}{l}\mathrm{F} \\
?\end{array}$ & E & 0.8 \\
\hline RZ Ari & 3305 & 145 & $\begin{array}{l}37.7 \\
56.5\end{array}$ & $\begin{array}{l}2 \\
1\end{array}$ & $\begin{array}{l}2 \mathrm{H} \\
1 \mathrm{H}\end{array}$ & $\overline{1.50-51}$ & 1.4 \\
\hline 4 Ori & $(\mathrm{S} 3.5 / 1)$ & - & $\begin{array}{l}36.0 \\
52.6 \\
74.1\end{array}$ & $\begin{array}{l}1 \\
2 \\
3\end{array}$ & $\begin{array}{l}3 \mathrm{H}: \\
2 \mathrm{H}: \\
1 \mathrm{H}:\end{array}$ & $\begin{array}{l}\overline{1.43-1.46} \\
1.40-1.41\end{array}$ & - \\
\hline UW Lyn & 3568 & 138 & $\begin{array}{l}26.0 \\
37.6 \\
49.5\end{array}$ & $\begin{array}{l}3 \\
1 \\
1\end{array}$ & $\begin{array}{l}3 \mathrm{H} \\
2 \mathrm{H} \\
1 \mathrm{H}\end{array}$ & $\begin{array}{l}\overline{1.29-1.45} \\
1.32-1.47\end{array}$ & $1.2-1.5$ \\
\hline$\mu \mathrm{Gem}$ & 3620 & 101 & $\begin{array}{l}20.0 \\
27.0 \\
51.0\end{array}$ & $\begin{array}{l}2 \\
1 \\
2\end{array}$ & $\begin{array}{l}2 \mathrm{H}: \\
1 \mathrm{H}: \\
\mathrm{F}:\end{array}$ & $\begin{array}{l}-\overline{1.35-1.35} \\
1.89-1.89\end{array}$ & 1.9 \\
\hline V523 Mon & 3439 & 110 & $\begin{array}{l}26.0 \\
34.1 \\
45.6\end{array}$ & $\begin{array}{l}2 \\
1 \\
2\end{array}$ & $\begin{array}{l}3 \mathrm{H} \\
2 \mathrm{H} \\
1 \mathrm{H}\end{array}$ & $\begin{array}{l}\overline{1.26-30} \\
1.35-38\end{array}$ & 0.8 \\
\hline $\mathrm{BC} \mathrm{CMi}$ & 3495 & 71 & $\begin{array}{l}20: \\
28: \\
45:\end{array}$ & $\begin{array}{l}2 \\
1 \\
3\end{array}$ & $\begin{array}{l}2 \mathrm{H} \\
1 \mathrm{H} \\
\mathrm{F}\end{array}$ & $\begin{array}{l}\overline{1.35-38} \\
1.56-60\end{array}$ & 0.7 \\
\hline UX Lyn & 3328 & 133 & $\begin{array}{l}37.2 \\
51.3\end{array}$ & $\begin{array}{l}1 \\
2\end{array}$ & $\begin{array}{l}2 \mathrm{H} \\
1 \mathrm{H}\end{array}$ & $\overline{1.38-42}$ & 1.1 \\
\hline FS Com & 3418 & 136 & $\begin{array}{l}38.2 \\
55.4\end{array}$ & $\begin{array}{l}2 \\
1\end{array}$ & $\begin{array}{l}2 \mathrm{H} \\
1 \mathrm{H}\end{array}$ & $\overline{1.45-46}$ & 1.1 \\
\hline V642 Her & 3513 & 91 & $\begin{array}{l}25.6 \\
35.7\end{array}$ & $\begin{array}{l}2 \\
1\end{array}$ & $\begin{array}{l}2 \mathrm{H} \\
1 \mathrm{H}\end{array}$ & $1.38-1.39$ & 0.8 \\
\hline R Lyr & 3455 & 178 & $\begin{array}{l}45.9 \\
64.1\end{array}$ & $\begin{array}{l}2 \\
1\end{array}$ & $\begin{array}{l}3 \mathrm{H} \\
2 \mathrm{H}\end{array}$ & $\overline{1.34-1.40}$ & 0.9 \\
\hline$\delta$ Sge & (II) & - & $\begin{array}{l}45.8 \\
66.7\end{array}$ & $\begin{array}{l}1 \\
2\end{array}$ & $\begin{array}{l}2 \mathrm{H}: \\
1 \mathrm{H}:\end{array}$ & $\overline{1.46-1.49}$ & - \\
\hline
\end{tabular}


cal models are somewhat dated and sparse, and they were intended for cooler, larger-amplitude variables such as Miras. Also, the models do not include radial pulsation modes higher than the second overtone; such modes are observed in a few stars. A new denser grid of models would therefore be useful.

\section{Long Secondary Periods}

At least one-third of our 77 PRGs have long secondary periods, an order of magnitude longer than the dominant radial periods. These long secondary periods have been found in samples of larger-amplitude red semi-regular variables, as well as in many survey samples in other Local Group galaxies. Wood (2000) has discussed the possible causes of these periods, and several authors (including Wood) have discussed this "problem" at this Colloquium. One of the stars in Table I EG And - is a well-known binary. The long secondary photometric period is half the orbital period, and is about 10 times the dominant radial period, so a binary explanation is promising in this case.

\section{Conclusions}

Our studies of bright PRGs provide an excellent model for the analysis and interpretation of the thousands of PRGs being discovered in surveys of Local Group galaxies. Hundreds of such PRGs must be multiperiodic; star-by-star analysis of these can determine their "pulsation mass"; their pulsation mode can be deduced from which sequence they inhabit in the period-luminosity relation the famous "Wood diagram" which was shown so many times at this Colloquium.

Acknowledgments. JRP thanks NSERC Canada for a research grant. Astronomy with automated telescopes at Tennessee State University is supported by NASA and by the National Science Foundation.

\section{References}

Dumm, T., Schild, H. 1998, NewA, 3, 137

Fox, M.W., Wood, P.R. 1982, ApJ, 259, 198

Koen, C., Laney, D. 2000, MNRAS, 311, 636

Ostlie, D.A., Cox, A.N. 1986, 311, 864

Percy, J.R., Desjardins, A., Yu, L., Landis, H.J. 1996, PASP, 108, 139

Percy, J.R., Wilson, J.B., Henry, G.W. 2001, PASP, 113, 983

Percy, J.R., Hosick, J. 2002, MNRAS, 334, 669

Percy, J.R., Besla, G., Velocci, V., Henry, G.W. 2003, PASP, 115, 479

Percy, J.R., Bakos, A.G. 2003, in The Garrison Festschrift, ed. R.O. Gray, C.J.

Corbally \& A.G.D. Philip (Schenectady: L. Davis Press), in press

Sperl, M. 1998, Comm. Asteroseismology (U. Vienna), \#112

Wood, P.R. 2000, Publ. Astron. Soc. Australia, 17, 8

Xiong, D.R., Deng, L., Cheng, Q.L. 1998, ApJ, 499, 355 


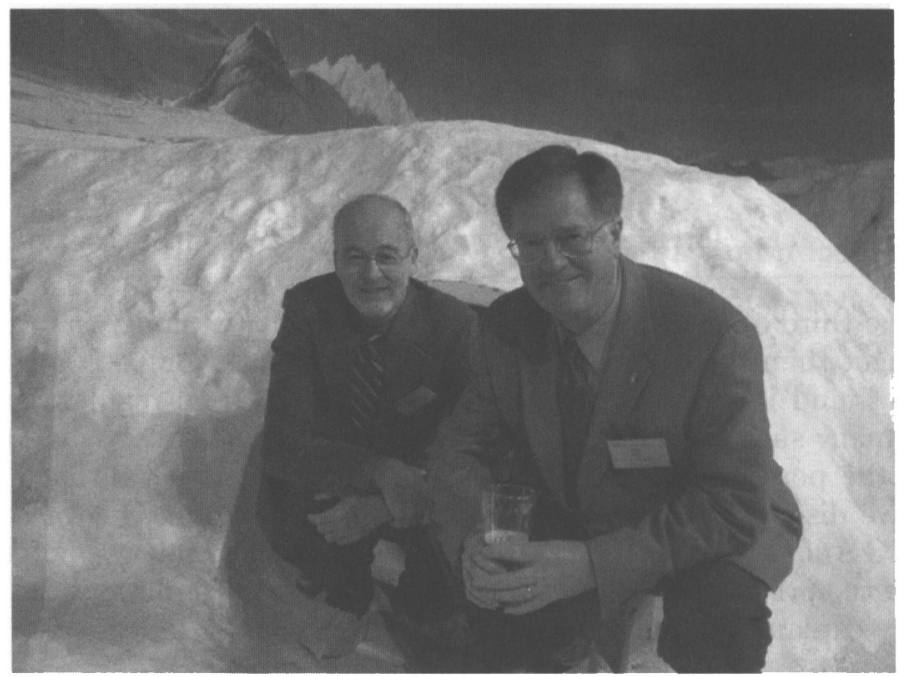

Michael Snowden and John Percy

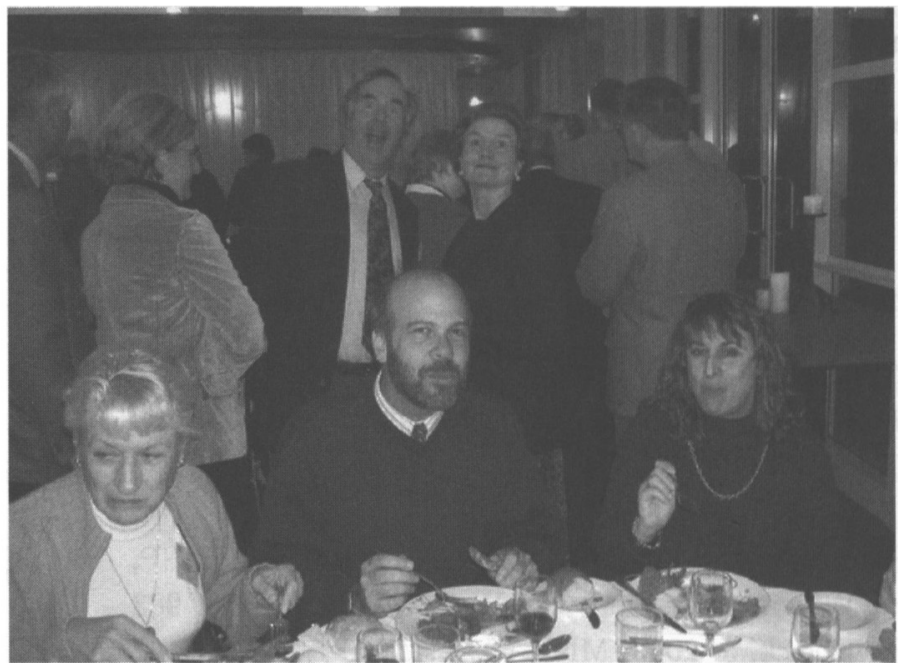

Janet Rountree, Steve and Leslie Kawaler, John and Vicky Hearnshaw 